CONTEMPORARY

ISSUES IN

NEUROLOGIC

PRACTICE

\title{
"Undiagnosing" multiple sclerosis
}

\section{The challenge of misdiagnosis in MS}

Andrew J. Solomon, MD

Eran P. Klein, MD, PhD

Dennis Bourdette, MD

Correspondence \& reprint requests to Dr. Solomon: andrew.solomon@uvm.edu

Editorial, page 1904

See page 1981

Supplemental data at www.neurology.org

Supplemental Data

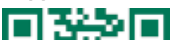

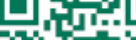

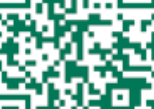
प)

\section{ABSTRACT}

Objective: To describe the clinical characteristics of encounters with patients misdiagnosed with multiple sclerosis (MS).

Methods: A cross-sectional Internet-based physician survey of MS specialists was performed.

Results: The response rate for the survey was 50.4\%. Of those who responded, the majority (95\%) reported having evaluated 1 or more patients who had been diagnosed with MS, but who they strongly felt did not have MS, within the last year. The majority of respondents (>90\%) also reported the use of disease-modifying therapy in a proportion of these patients. Most respondents (94\%) found clinical encounters with these patients equally or more challenging than giving a new diagnosis of MS. Fourteen percent of respondents reported that they did not always inform such patients of their opinion that they did not have MS.

Conclusions: The misdiagnosis of MS is common and has significant consequences for patient care and health care system costs. Caring for a patient with a misdiagnosis of MS is challenging, and at times honest disclosure of a misdiagnosis represents an important ethical concern for neurologists. More data are needed on this patient population to improve diagnostic acumen and the care of these patients. Neurology ${ }^{\circledR} 2012 ; 78: 1986-1991$

\section{GLOSSARY}

DMT = disease-modifying therapy; $\mathbf{M S}=$ multiple sclerosis .

The challenge of giving a diagnosis of multiple sclerosis (MS) has been well documented. Established diagnostic criteria, along with clinical, radiologic, and laboratory assessments, are relied upon to confer a diagnosis of $\mathrm{MS} .{ }^{1}$ However, a number of diseases and syndromes may mimic MS, ${ }^{2}$ and as a result neurologists may encounter the clinical challenge of having to undo a misdiagnosis of MS. While little objective data are available on the prevalence of the misdiagnosis of MS, it has important clinical, psychosocial, and economic consequences for the patient, treating neurologist, and health care system at large.

A survey of MS specialists was conducted to determine how often MS specialists encounter patients with an erroneous diagnosis of MS and to assess the clinical characteristics of these patients. Physician responses to these encounters were explored. Correlations between demographic, educational, and practice characteristics were investigated. The results of this study demonstrate not only that MS specialists commonly encounter patients misdiagnosed with MS, and that many of these patients are taking inappropriate and expensive pharmacotherapy, but also that some clinicians experience the decision to "undiagnose" MS to be ethically challenging.

From the Department of Neurology (A.J.S.), Fletcher Allen Heath Care, University Health Center, University of Vermont College of Medicine, Burlington; Department of Neurology (F.P.K., D.B.), Oregon Health \& Science University, Portland; and Neurology Service (F.P.K., D.B.) and MS Center of Excellence-West (D.B.), Department of Veterans Affairs Medical Center, Portland, OR.

Study funding: This publication was made possible with support from the Oregon Clinical and Translational Research Institute, grant number UL1 RR024140 from the National Center for Research Resources, a component of the NIH, and NIH Roadmap for Medical Research. This study was funded in part by the Partners Multiple Sclerosis Fellowship Award. Dr. Solomon was a Department of Veterans Affairs Special Fellow in Multiple Sclerosis, Dr. Klein is a Department of Veterans Affairs Special Fellow in Geriatric Neurology, and Dr. Bourdette receives research funding from the Department of Veterans Affairs Office of Research and Development.

Go to Neurology.org for full disclosures. Disclosures deemed relevant by the authors, if any, are provided at the end of this article. 
METHODS A physician survey focused on patients misdiagnosed with MS was developed. This survey was not a previously validated instrument, nor was it formally validated prior to the use in this study. During development, questions and responses were reviewed by colleagues in the field of neurology and MS as well as faculty of programs at the Oregon Clinical and Translational Research Institute, a NIH Clinical and Translational Science Award center. The survey was made available for respondents on the Internet using the services of surveymonkey. com, and may be viewed in appendix e- 1 on the Neurology ${ }^{\circledR}$ Web site at www.neurology.org.

Neurologists in private and academic practices focused on the care of patients with MS in the United States and Canada were identified through the Consortium of MS Centers Web site at designated "centers" and through use of google.com. Public institutional e-mail addresses for neurologists at academic centers identified by this search were compiled through a search of university Web sites and article publications. An additional few e-mail addresses for neurologists in private practice MS centers were also obtained.

An invitation to participate in the study with an Internet Web address link to the survey was e-mailed to 242 individual neurologists. Respondents were given 2 weeks to complete the survey. An e-mail reminder was sent 1 week after the initial invitation.

The design of the survey prevented the completion of more than 1 survey per computer. Responses were collected from surveymonkey.com, imported to Microsoft Excel, and analyzed using SAS 9.22 software. Descriptive statistics such as number and percentage were generated for categorical variables. Fisher exact test and $\chi^{2}$ were used to determine relationships between categorical variables.

Standard protocol approvals, registrations, and patient consents. The study and survey were reviewed and approved by the OHSU institutional review board. Responses were anonymous and deidentified.

RESULTS Respondent demographics. Invitations to participate in the study were e-mailed to 242 individual neurologists and 122 surveys were completed, resulting in a response rate of $50.4 \%$. The individual and practice characteristics of respondents who completed the survey instrument are detailed in table 1 . Since the survey used categorical rather than continuous variables for responses, a mean and median are not reported. The majority of respondents, 101/122 $(82.8 \%)$, devoted more than half of their practice to the care of patients with MS. Approximately half $(48.3 \%)$ had completed training in neurology in the last 15 years and approximately half $(51.7 \%)$ in the last 16 years or greater. The largest subgroup of respondents had completed training 20 years or greater (40.2\%). Eighty (65.6\%) had completed a MS or neuroimmunology fellowship or postdoctoral training.

The majority of respondents currently practiced neurology in an academic institution $(90.2 \%)$ in the United States $(96.7 \%)$. Of those practicing in academic institutions, approximately half $(49.1 \%)$ reported that $51 \%$ or greater of their academic time was devoted to clinical care of patients. A minority of respondents (13.9\%) reported caring for patients full or part time at a Veterans Affairs medical center. The

\section{Table 1 Respondent demographics}

Characteristics

Practice devoted to MS

$<10 \%$

$1(0.82)$

$10 \%-25 \%$

10 (8.2)

$25 \%-50 \%$

10 (8.2)

$51 \%-75 \%$

19 (15.6)

$>75 \%$

82 (67.2)

Time since completion of neurology training

$<5 y$

17 (13.9)

5-10y

31 (25.4)

11-15y

11 (9)

16-20y

14 (11.5)

$>20 y$

49 (40.2)

MS fellowship

Yes

80 (65.6)

No

42 (34.4)

Practice setting

Academic

$110(90.2)$

Private/nonacademic

10 (8.2)

Other

2 (1.64)

In academic appointment, clinical care effort

$<10 \%$

2 (1.8)

$10 \%-25 \%$

27 (24.6)

$25 \%-50 \%$

27 (24.6)

$51 \%-75 \%$

28 (25.5)

$>75 \%$

26 (23.6)

Country

United States

118 (96.7)

Canada

4 (3.3)

US Veterans Affairs practice

Yes

17 (13.9)

No

105 (86.1)

Time, new consults

$30 \mathrm{~min}$

$1(0.8)$

$45 \mathrm{~min}$

11 (9)

$60 \mathrm{~min}$

74 (60.7)

$75 \mathrm{~min}$

14 (11.5)

$90 \mathrm{~min}$

21 (17.2)

$>90 \mathrm{~min}$

$1(0.8)$

Time, return visits

$15 \mathrm{~min}$

7 (5.7)

$30 \mathrm{~min}$

96 (78.7)

$45 \mathrm{~min}$

16 (13.1)

$60 \mathrm{~min}$

Abbreviation: MS = multiple sclerosis

majority reported 1 hour or greater allotted for new patient consultations (90.2\%), and half an hour or greater for return patient visits $(94.3 \%)$. 


\begin{tabular}{|c|c|c|}
\hline Table 2 & $\begin{array}{l}\text { Patients misdiagnosed with } \\
\text { multiple sclerosis }\end{array}$ & \\
\hline \multicolumn{2}{|c|}{ Characteristics } & No. (\%) \\
\hline \multicolumn{3}{|c|}{$\begin{array}{l}\text { Evaluated a misdiagnosed patient } \\
\text { within last year }\end{array}$} \\
\hline \multicolumn{2}{|l|}{ Yes } & $116(95.1)$ \\
\hline \multicolumn{2}{|l|}{ No } & $6(4.9)$ \\
\hline \multicolumn{3}{|c|}{ No. seen within last year } \\
\hline \multicolumn{2}{|l|}{$1-2$} & 30 (25.9) \\
\hline \multicolumn{2}{|l|}{$3-5$} & $46(39.7)$ \\
\hline \multicolumn{2}{|l|}{$6-10$} & 20 (17.2) \\
\hline \multicolumn{2}{|l|}{$\geq 10$} & 20 (17.2) \\
\hline \multicolumn{3}{|c|}{ Estimated on DMT } \\
\hline \multicolumn{2}{|l|}{$0 \%$} & $6(5.2)$ \\
\hline \multicolumn{2}{|l|}{$1-25 \%$} & 35 (30.2) \\
\hline \multicolumn{2}{|c|}{$26-50 \%$} & 28 (24.1) \\
\hline \multicolumn{2}{|c|}{$51-75 \%$} & $17(14.7)$ \\
\hline \multicolumn{2}{|l|}{$\geq 75 \%$} & 30 (25.9) \\
\hline
\end{tabular}

Abbreviation: DMT = disease-modifying therapy.

Patients misdiagnosed with MS. Respondents were asked: "Have you ever evaluated a patient who carried a diagnosis of MS (given by another provider) for longer than a year who, after your neurologic exam and review of lab data, you strongly felt did NOT in fact have MS?" Nearly all respondents (95.1\%) had evaluated such a patient in the past.

The phrasing "strongly felt did NOT in fact have MS" was designed to preclude patients for whom there was instead "uncertainty" regarding a diagnosis, a "suspected" alternative diagnosis, or patients who "may have MS" in the future. Instead, the survey questions were intended to query respondents on the characteristics of patients whom they strongly felt had a diagnosis other than MS given their evaluation. We use the terms "misdiagnosis" or "misdiagnosed" to refer to these patients.

Table 2 presents further data regarding these misdiagnosed patients. Within the preceding year approximately $40 \%$ of respondents estimated that they had seen 3-5 such patients. Over one-third (34.4\%) reported seeing 6 or more misdiagnosed patients in the last year, including $20(17.2 \%)$ respondents who had seen 10 or more such patients. To estimate the number of patients who had been seen with a misdiagnosis of MS, we used the median from the range of each individual response to this question and assigned the value of 10 for a response of " 10 or more," to estimate the total number of patients. Using this method, our respondents had seen an estimated 598 patients misdiagnosed with MS within the last year.

Respondents were asked to "estimate the percentage of these misdiagnosed patients who had been started on disease-modifying therapy for MS by another provider." Seventy-five (64.7\%) of the respondents estimated that more than a quarter $(\geq 26 \%)$ of all the misdiagnosed patients they had seen were on disease-modifying therapy (DMT). This included 30 (25.9\%) respondents who estimated $75 \%$ or more of the misdiagnosed patients they had seen were on DMT. Additional detailed data on the estimated frequency of misdiagnosed patients on DMT is seen in table 2. We used the median calculated above for the number of misdiagnosed patients seen by each respondent and then used the median from the response chosen by the same respondent for the estimated number of those patients on DMT (assigning $75 \%$ for a response of "equal or greater than $75 \%$ ”) to calculate the estimated number of misdiagnosed patients on DMT seen per respondent. In total, within the last year all respondents had seen an estimated 279 patients misdiagnosed with MS who were on DMT.

Respondents were asked: "The most likely alternative diagnosis in patients you have seen with a longstanding misdiagnosis (i.e., greater than 1 year) of MS have been (check all that apply): ...” and were allowed to choose more than one diagnosis among a list of 15 . There was also an option to choose "other" with completion of a response box for free text. In figure 1, the proportion of each diagnosis chosen per number of responses is displayed. One response indicating a particular alternative diagnosis may reflect several misdiagnosed patients seen by a respondent any time in the past. The frequency of each diagnosis reported therefore is not quantitative and does not represent the absolute number of misdiagnosed patients with an alternative diagnosis reported by respondents. These data may reflect the relative frequency of a particular suspected alternative diagnosis in patients misdiagnosed with MS.

Most respondents $(94,81 \%)$ chose nonspecific white matter abnormalities on brain MRI and small vessel ischemic disease $(91,78.5 \%)$ and half (58, $50 \%)$ reported migraine. A significant number reported psychiatric disease $(52,44.8 \%)$ and almost one-third reported fibromyalgia 36 (31\%). Neuromyelitis optica $(47,40.5 \%)$ also was reported frequently. The frequency of additional responses is seen in figure 1. Thirteen (11.2\%) also chose "other" and completed a response box with free text. These responses included celiac disease, focal dystonia, paraneoplastic disorder, orbital pseudotumor, HTLV-1 myelopathy, conversion disorder, cervical spondylosis with myelopathy, neurosarcoidosis, Susac syndrome, cerebellar ataxia, adult onset Alexander disease, and CMT-2. 
Figure 1 Suspected alternative diagnoses in patients misdiagnosed with multiple sclerosis (MS)

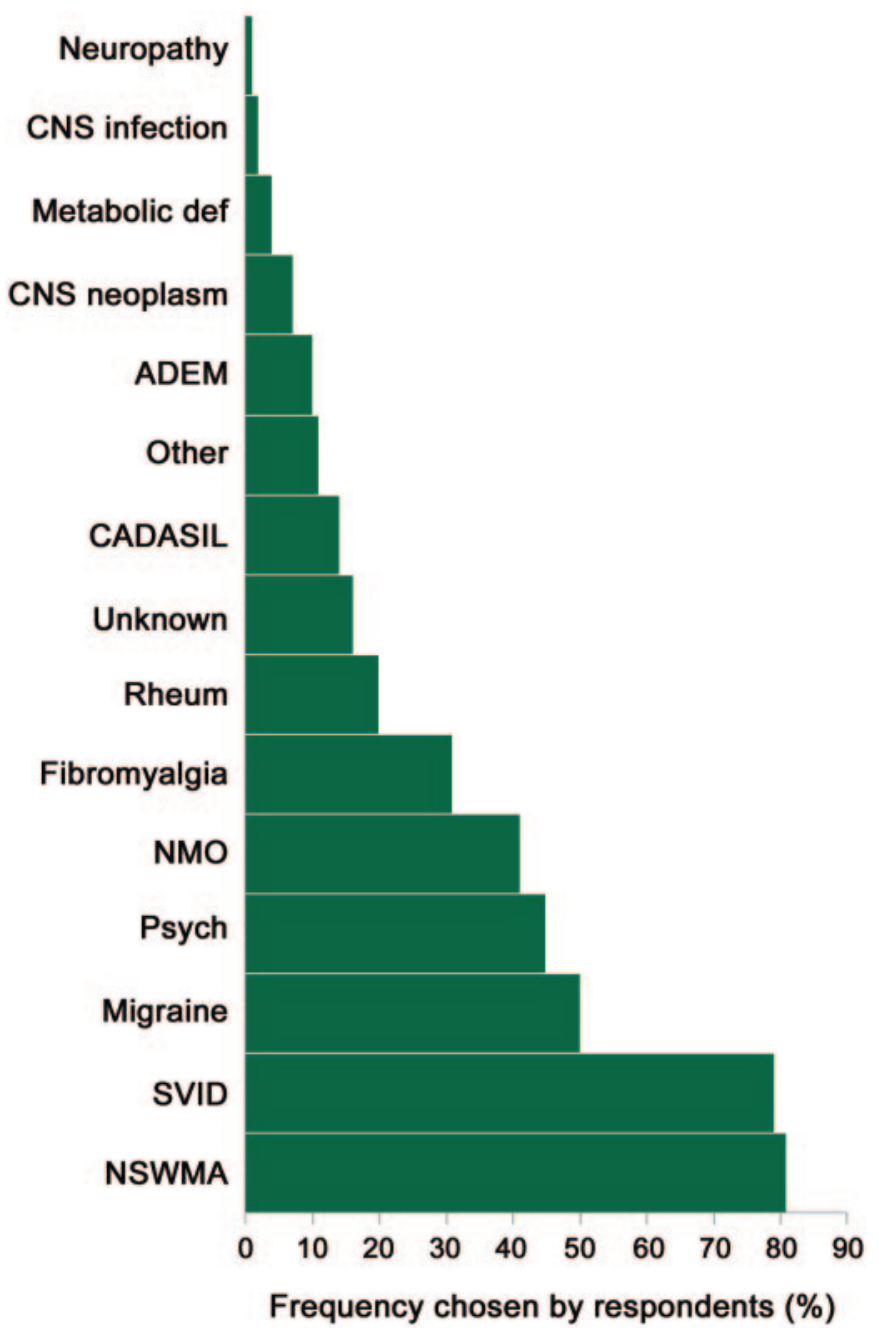

Respondents were asked to select from a list of 15 suspected alternative diagnoses in patients misdiagnosed with MS whom they had evaluated at any time in the past. The proportion (\%) of respondents who chose each diagnosis is presented. Respondents were allowed to choose more than one diagnosis. Each selection may reflect multiple misdiagnosed patients-these data do not represent the absolute number of misdiagnosed patients. $\mathrm{ADEM}=$ acute disseminated encephalomyelitis; CADASIL = cerebral autosomal dominant arteriopathy with subcortical infarcts and leukoencephalopathy; $\mathrm{NMO}=$ neuromyelitis optica; NSWMA = nonspecific white matter abnormalities; SVID = small vessel ischemic disease.

Informing of misdiagnosis. The majority of respondents $(77,66.4 \%)$ stated that informing a patient with a long-established diagnosis of MS that they likely do not have MS was more challenging than informing a patient of a new diagnosis of MS. A significant number of respondents felt that both clinical situations were equally challenging $(32,27.6 \%)$. Seven $(6 \%)$ believed that informing a patient of a new diagnosis was more challenging than a misdiagnosis.

After evaluating a patient who they strongly believed did not have MS, 16 (13.8\%) respondents reported that they sometimes had chosen not to inform such patients of their suspicion regarding the misdi- agnosis. These respondents were asked for reasons important to their decision not to inform of the misdiagnosis in the past, and were allowed to choose either yes or no for 6 provided responses. Figure 2 presents these data regarding a decision not to inform patients of a suspected misdiagnosis. The absence of DMT and resulting medication-associated risks affected this decision in 11 (68.8\%). Ten (62.5\%) replied that the risk for psychological harm from a change to perceived diagnosis was important to their decision not to inform.

Nine $(56.3 \%)$ replied that the presence of a suspected benign alternative diagnosis that did not require additional treatment was a reason they had chosen not to inform of a misdiagnosis of MS in the past. Eight (50\%) believed that a change in diagnosis was likely to lead to a loss of social support or support network and this was important to their decision not to inform. Six $(37.5 \%)$ responded that likely financial harm due to change in eligibility for benefits was important for their decision. Six (37.5\%) believed that the change in diagnosis should be left to the referring physician aided by a consultation letter.

Associations with informing of misdiagnosis. Having chosen not to inform a patient of a misdiagnosis of MS was not significantly associated with the proportion of practice dedicated to MS, academic or private practice setting, work at the US Department of Veterans Affairs, proportion of time devoted to clinical care in an academic position, or less than 60 minutes for new consultations and less than 30 minutes for return visits.

Those having completed postdoctoral training or a fellowship in neuroimmunology or MS were more likely to have decided not to inform a patient of a misdiagnosis in the past $(p=0.046)$. Completion of neurology training within the last 10 years was also associated with having chosen not to inform a patient of a suspected misdiagnosis $(p=0.044)$.

Indicating that either informing a patient of a misdiagnosis of MS was more challenging than informing a patient of a new diagnosis of MS or that both were equally challenging was not significantly associated with proportion of practice dedicated to MS, academic or private practice setting, work at the US Department of Veterans Affairs, proportion of time devoted to clinical care in an academic position, time since completion of neurology training, having completed postdoctoral or fellowship training, or having less than 60 minutes for new consultations or less than 30 minutes for return visits.

DISCUSSION The results of this study indicate that MS specialists commonly encounter patients who they believe have been misdiagnosed with MS. Re- 


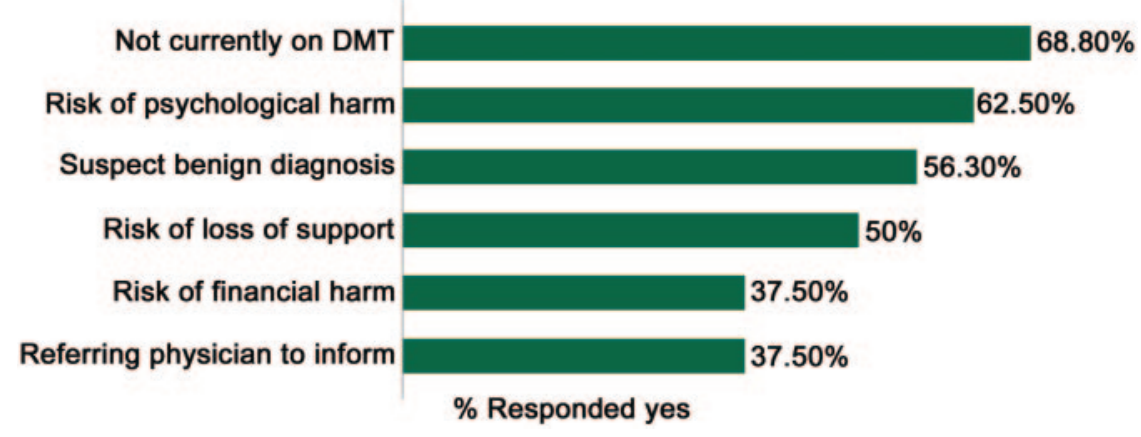

The 16 respondents who indicated that they sometimes did not inform patients of a misdiagnosis of MS were asked if 6 reasons were important to this decision. The proportion (\%) of these respondents who answered affirmatively to the importance of these reasons in their decision not to inform is presented. DMT = disease-modifying therapy.

spondents reported a number of suspected alternative diagnoses in these cases, many of which may reflect overreliance on MRI criteria for diagnosis. Misdiagnosed patients are subject to a number of risks, including DMT. At times some MS specialists chose not to inform patients of a misdiagnosis, and almost all found such a clinical encounter to be equally or more challenging than giving a new diagnosis of MS

Inappropriate use of imaging criteria for diagnosis $^{1,3}$ may have played an important role in misdiagnosis in many of the cases reported by respondents. The frequency of suspected alternative diagnoses of small vessel ischemic disease, nonspecific white matter abnormalities, and migraine reported in patients misdiagnosed with MS likely reflects improper interpretation of MRI findings. Data supporting therapeutic benefit from early initiation of DMT in MS generates an imperative to diagnose MS or clinically isolated syndrome early and begin therapy. ${ }^{4,5}$ Overreliance on MRI findings in patients with syndromes for which established MS diagnostic imaging criteria have not been validated, rather than prudent clinical and laboratory monitoring, may have resulted in misdiagnosis in many cases.

Misdiagnosis of MS carries potential for serious harm. Unrecognized and inappropriately treated, $\mathrm{NMO}$ and cervical spondylosis can result in irreversible disability. Misdiagnosis of nonspecific MRI changes, small vessel ischemic disease, or "pseudoMS"6 may confer significant psychosocial harm. DMT use in the absence of MS exposes patients not only to unnecessary health risks but also financial costs. Presuming a cost of $\$ 40,000$ USD/year for standard DMT, the estimated 279 misdiagnosed patients on DMT evaluated by our respondents would have incurred \$11 million in medical expense for the last year. If total medical care, nonmedical indirect costs, and additional years of misdiagnosis are considered, these costs are substantially higher. The pos- sible participation of these patients in clinical trials for new MS therapies confers additional unnecessary medical risk and has the potential to confound resulting data and lead to erroneous interpretation of results.

Most neurologists surveyed chose to correct misdiagnoses of MS, but not all did. The institutional and psychological barriers to discussion of mistakes in medicine are well known. ${ }^{7}$ The difficulty for most, and the reluctance of some surveyed neurologists to inform patients of a misdiagnosis of MS embodies a common tension in medical practice: between supporting patient autonomy and promoting beneficent goals. ${ }^{8}$ Clinicians were motivated to correct misdiagnoses and allow patients to make accurate and autonomous decisions about their health care as well as by a desire to protect patients from harms that might attend a change in diagnosis. This desire to shield patients from harm seemed to extend beyond a claim of "therapeutic privilege" - that patients might suffer psychologically from full disclosure9-and to broader concerns for social and economic harms. The results of this study suggest that neurologist decision-making in this area is complex and nuanced, and deserves further study.

There were several limitations to this study and results should be interpreted cautiously. Our survey instrument has not been validated, and $50 \%$ of the neurologists who were invited chose not to participate in the study. It is possible that a number of the neurologists surveyed, while identified as caring for MS patients via the Web site of their academic institution, did not in fact have extra training or expertise in MS. The study design was subject to temporal bias, recall bias, and selection bias. The data are also primarily limited to the experience of academic neurologists in the United States. It is possible that some respondents may have misunderstood the survey questions regarding misdiagnosed patients to include 
consideration of all patients who do not meet strict diagnostic criteria for clinically definite MS. Although not the focus of our study, this may therefore include responses reflecting patients who remain at high risk for subsequent development of MS (i.e., clinically isolated syndrome) rather than other alternative diagnoses. It also remains possible that a number of the patients whom our respondents strongly believed did not have MS may ultimately come to be diagnosed with MS after subsequent monitoring and evaluation.

The apparent frequency of patients misdiagnosed with MS and the number of these patients receiving DMT found by this study are concerning. Caring for these patients is challenging, and at times the honest disclosure of a misdiagnosis represents an important ethical concern for neurologists. Prospective quantitative data on the characteristics of these patients, their correct diagnoses, and resulting patientphysician encounters is needed. Future research must also consider the impact of newly approved diagnostic imaging criteria ${ }^{1}$ for MS as well as the implications of the increasingly recognized radiologically isolated syndrome ${ }^{10}$ on misdiagnosis. Further study will help us to improve our diagnostic acumen, recognize our mistakes, and learn how to better communicate them to our patients.

\section{AUTHOR CONTRIBUTIONS}

Andrew Jay Solomon: drafting/revising the manuscript, study concept or design, analysis or interpretation of data, acquisition of data, study supervision. Eran Patrick Klein: drafting/revising the manuscript, study concept or design. Dennis Bourdette: drafting/revising the manuscript, study supervision.

\section{DISCLOSURE}

Dr. Solomon reports no disclosures. Dr. Klein received an honorarium for speaking at the AAN annual meeting in 2011. Dr. Bourdette has research grants from the NIH, the Department of Veterans Affairs, and the National Multiple Sclerosis Society and has received educational grants and speaker honoraria from Teva Neuroscience, Biogen Idec, and EMD Serono, Inc. Go to Neurology.org for full disclosures.

Received August 2, 2011. Accepted in final form December 14, 2011.

\section{REFERENCES}

1. Polman $\mathrm{CH}$, Reingold SC, Banwell B, et al. Diagnostic criteria for multiple sclerosis: 2010 revisions to the McDonald criteria. Ann Neurol 2011;69:292-302.

2. Miller DH, Weinshenker BG, Filippi M, et al. Differential diagnosis of suspected multiple sclerosis: a consensus approach. Mult Scler 2008;14:1157-1174.

3. Polman CH, Reingold SC, Edan G, et al. Diagnostic criteria for multiple sclerosis: 2005 revisions to the "McDonald Criteria." Ann Neurol 2005;58:840-846.

4. Bates D. Treatment effects of immunomodulatory therapies at different stages of multiple sclerosis in short-term trials. Neurology 2011;76(suppl 1):S14-S25.

5. Coyle PK. Early treatment of multiple sclerosis to prevent neurologic damage. Neurology 2008;71(suppl 3):S3-S7.

6. Paulson GW. Pseudo-multiple sclerosis. South Med J 1996;89:301-304.

7. Institute of Medicine. To Err is Human; Building a Safer Health System. Washington, DC: National Academy Press; 1999.

8. Beauchamp TL, Childress JF. Principles of Biomedical Ethics, vol. 6. New York: Oxford University Press; 2009.

9. President's Commission for the Study of Ethical Problems in Medicine and Biomedical and Behavioral Research. Making Health Care Decisions 1, Report. Washington, DC: US Government Printing Office; 1982.

10. Spain R, Bourdette D. The radiologically isolated syndrome: look (again) before you treat. Curr Neurol Neurosci Rep 2011;11:498-506. 